\title{
Combined Detection of Serum IL-10, IL-17, and CXCL10 Predicts Acute Rejection Following Adult Liver Transplantation
}

\author{
Nayoung Kim ${ }^{1, *}$, Young-In Yoon ${ }^{2,3}$, Hyun Ju Yoo ${ }^{1}$, Eunyoung Tak ${ }^{1}$, Chul-Soo Ahn ${ }^{2}$, Gi-Won Song ${ }^{2}$, \\ Sung-Gyu Lee ${ }^{2}$, and Shin Hwang ${ }^{2, *}$
}

\begin{abstract}
Discovery of non-invasive diagnostic and predictive biomarkers for acute rejection in liver transplant patients would help to ensure the preservation of liver function in the graft, eventually contributing to improved graft and patient survival. We evaluated selected cytokines and chemokines in the sera from liver transplant patients as potential biomarkers for acute rejection, and found that the combined detection of IL-10, IL-17, and CXCL10 at 1-2 weeks post-operation could predict acute rejection following adult liver transplantation with $97 \%$ specificity and $94 \%$ sensitivity.
\end{abstract}

\section{INTRODUCTION}

Liver transplantation is often the only option for otherwise nontreatable chronic and acute liver failure. Although the development of immunosuppressive regimens and surgical techniques in last few decades has tremendously improved graft and patient survival, acute rejection still causes serious problems in $10-20 \%$ of liver transplant (LT) patients (Song et al., 2014). Standard diagnosis includes liver biopsy, which itself is a highly risky procedure. Hence, there is an urgent need for noninvasive predictive and diagnostic biomarkers to detect acute rejection following LT. As acute rejection is a result of cellular immune responses, circulating cytokines and chemokines could serve as useful predictive or diagnostic biomarkers for acute rejection in LT patients. In particular, cytokines and chemokines that are involved in inflammation and $T$ cell function could be potential biomarkers, as acute rejection is a T cell-

\footnotetext{
${ }^{1}$ Department of Convergence Medicine \& Asan Institute for Life Sciences, ${ }^{2}$ Division of Liver Transplantation and Hepatobiliary Surgery, Department of Surgery, Asan Medical Center, University of Ulsan College of Medicine, Seoul 05505, Korea, ${ }^{3}$ Department of Hepatobiliary Pancreas Surgery, Korea University Anam Hospital, Seoul 02841, Korea

*Correspondence: naykim@amc.seoul.kr (NK); shwang@amc.seoul.kr (SH)
}

Received 26 May, 2016; revised 18 July, 2016; accepted 20 July, 2016; published online 5 August, 2016

Keywords: acute rejection, biomarker, chemokine, cytokine, liver transplant mediated process.

Many cytokines and chemokines have been investigated as potential biomarkers for the diagnosis of graft rejection or dysfunction following liver transplantation. Microarray analysis has shown that interleukin (IL)-3, tumor necrosis factor-alpha (TNF$\alpha$ ), and $I L-10$ mRNAs are increased in biopsy samples from $L T$ patients that show acute rejection (Tannapfel et al., 2001). Another study demonstrated that $I L-2$ and TNF- $\alpha$ mRNAs were upregulated in biopsy samples collected at day 21 postoperation (PO) from $\mathrm{LT}$ patients with acute rejection (Sreekumar et al., 2002). These genes were the only cytokines found to be differentially expressed among several genes analyzed in each study. $I L-2, I L-4$, and $I L-15$ mRNAs increased in peripheral blood mononuclear cells from renal allograft patients in the pre-rejection phase (Lee et al., 2012). In a rat liver allograft model, inhibition of IL-4 by mycophenolate was suggested to inhibit rejection (Huang et al., 2003). Serum interferongamma (IFN- $\gamma$ ) peri-operationally, and IL-10 and CXCL10 on days 1 and 2 PO were suggested as biomarkers for early allograft dysfunction after liver transplantation (Karakhanova et al., 2016). IL-17 and IL-23 increased in the sera of patients with rejection on days 1 and 7 PO (Fabrega et al., 2009). Intracellular IFN- $\gamma$ and IL-2 levels significantly changed in LT patients with acute rejection at 1 week $\mathrm{PO}$, although soluble IL-2 levels were not affected (Millan et al., 2013). Soluble IL-17 in the supernatant after $48 \mathrm{~h}$ culture of whole blood cells with concanavalin $\mathrm{A}$ and the percentages of IFN- $\gamma+\mathrm{CD} 4+\mathrm{CD} 69+$ and IFN$\gamma+\mathrm{CD} 8+\mathrm{CD} 69+$ cells are considered predictive biomarkers of acute rejection in the $2^{\text {nd }}$ and $4^{\text {th }}$ weeks PO (Millan et al., 2014). Although IL-6 is considered to be involved in rejection in lung transplantation (Nakagiri et al., 2012), pre-operational IL-6 levels are lower in LT patients with early allograft dysfunction (Friedman et al., 2012).

CXCR3 ligands have been suggested as candidates for the early diagnosis of acute rejection in various organs such as the kidney, liver, and heart. CXCR3 is expressed on activated T cells and natural killer cells, and its ligands are CXCL9 (MIG), CXCL10 (IP-10), and CXCL11 (I-Tac) (Capece and Kim, 2016). A long-term follow-up study of LT patients for up to 7 years suggested CXCL10 as a biomarker to predict fibrosis in the first year after LT for hepatitis C infection, whereas CXCL11 was less useful (Berres et al., 2011). Serum CXCL9 and CXCL10 levels were found to be significantly higher in LT patients with early allograft dysfunction on day $1 \mathrm{PO}$, but not after that 
IL-10, IL-17, and CXCL10 Predict Acute Liver Rejection

Nayoung Kim et al.

Table 1. Demographic and clinical characteristics of liver transplant patients

\begin{tabular}{|c|c|c|}
\hline & Control & Rejection \\
\hline Gender & M 27, F 8 & $\mathrm{M} 13, \mathrm{~F} 4$ \\
\hline Age (years) & $51 \pm 1.191$ & $48 \pm 2.008$ \\
\hline Type of operation & LD 31, CD 3, dual 1 & LD 15, CD 1, dual 2 \\
\hline ABO compatibility & C 27, I 8 & C 14,13 \\
\hline \multirow[t]{8}{*}{ Original diseases } & LC (HBV) 26 & LC (HBV) 6 \\
\hline & LC (HBV, alcohol) 1 & LC (HCV) 2 \\
\hline & LC (NBNC) 1 & PSC 1 \\
\hline & ALC LC 4 & $\mathrm{HCC} 1$ \\
\hline & SBC 1 & ALC LC 3 \\
\hline & Retransplant 2 & Toxic hepatitis-FHF 1 \\
\hline & & Fulminant hepatitis (r/o HAV AH) 1 \\
\hline & & Retransplant 2 \\
\hline Days to rejection & & $18 \pm 7.295$ \\
\hline Cold ischemic time & $83.5 \pm 11.564$ & $96 \pm 16.262$ \\
\hline Warm ischemic time & $38 \pm 2.718$ & $38 \pm 3.572$ \\
\hline Total ischemic time & $124 \pm 12.322$ & $136 \pm 18.036$ \\
\hline MELD score & $12 \pm 1.799$ & $15.5 \pm 1.743$ \\
\hline
\end{tabular}

$\mathrm{AH}$, acute hepatitis; ALC, alcoholic; C, compatible; CD, cadaveric donor; dual, dual transplantation; FHF, fulminant hepatic failure; HAV, hepatitis A virus; HBV, hepatitis B virus; HCC, hepatocellular carcinoma; HCV, hepatitis C virus; I, incompatible; LC, liver cirrhosis; LD, living donor; NBNC, non-HBV and non-HCV; PSC, primary sclerosing cholangititis; SBC, secondary biliary cirrhosis

(Friedman et al., 2012). A recent report suggested that reduced CD44 and increased CXCL9 at day 1 PO could be noninvasive biomarkers in the sera from LT patients with rejection (Raschzok et al., 2015).

Despite these previous efforts, there are no reliable candidate biomarkers for clinical application. In addition, expression of cytokines and chemokines is influenced by several factors such as infections and autoimmune diseases. Hence, the combination of diagnostic and predictive biomarkers could improve the sensitivity and specificity of biomarkers. Accordingly, the aim of this study was to investigate serum levels of selected cytokines and chemokines weekly for one month after adult liver transplantation to evaluate the possibility of using a combination of diagnostic and predictive biomarkers.

\section{MATERIALS AND METHODS}

\section{Patients}

From February 2012 to July 2012, adult patients undergoing LT surgery at Asan Medical Center, Seoul, Korea were prospectively enrolled in this study. The study was approved by the Institutional Review Board (Approval No. 2011-0898) and written informed consent was obtained from all participants. All patients were provided with standard care and treatment. The demographic and clinical characteristics of the patients are shown in Table 1.

Serum collection and selection

Whole blood from LT recipients at LT wards, not at the intensive care unit or outpatient clinics, was collected in STT BD Vacutainer tubes (BD, USA) weekly for 4 weeks $\mathrm{PO}$, and sera were harvested within $2 \mathrm{~h}$ and stored in a deep freezer until use. Six months later, the patients were classified into acute rejection and control (no rejection) groups by reviewing medical records.
A clinical diagnosis of acute rejection was considered when alanine aminotransferase (ALT) and bilirubin levels rose without evidence of infections, thrombosis, and other complications. Biopsy confirmed the results; in some cases, the patients were immediately treated with standard rejection therapeutics without biopsy, which was considered as clinical rejection. Patients without rejection of similar ages and gender were selected as the control group. Among the 164 patients enrolled, 17 patients with rejection and 35 controls were selected; however, given that it was not possible to collect samples in a timely manner for all the patients at the designated dates, the sample numbers vary at each time point. None of the patients had other complications or infections at one month PO.

Multiplex chemokine assay

A multiplex chemokine assay to assess serum concentration of IL-2, IL-4, IL-6, TNF- $\alpha$, IFN- $\gamma$, IL-10, IL-17A, CXCL9, CXCL10, and CXCL11 was performed using a Cytometric Bead Array system (BD Biosciences, USA) following the manufacturer instructions. The results were analyzed with FACScanto II (BD Biosciences) and FCAP Array multiplex analysis software (BD Biosciences).

\section{Statistical analysis}

Medians and standard errors of the means were calculated with GraphPad InStat 3 (Version 3.10) software (GraphPad, Inc., USA). Two-tailed Student $t$ tests were performed with Excel software (Microsoft, Co., USA). Receiver operating characteristic (ROC) curve analysis was performed to estimate the potential predictive or prognostic value of the parameters investigated using area under the curve (AUC) estimation with SPSS software (SPSS, Inc., USA). Multivariate analysis and logistic regression analysis were also performed using SPSS software. Missing data were not replaced or imputed. A value of $P<0.05$ 
Table 2. Summary of the predictive capacity for acute rejection

\begin{tabular}{lcccc}
\hline & $\mathrm{IL}-17(1$ week PO) & IL-10 (2 weeks PO) & CXCL10 (1 week PO) & Multivariate modeling \\
\hline AUC & 0.750 & 0.729 & 0.752 & 0.949 \\
Sensitivity & 75 & 81 & 78 & 94 \\
Specificity & 70 & 75 & 77 & 97 \\
Cut-off values & 24.56 & 18.31 & 217.00 & \\
\hline
\end{tabular}

AUC, area under the curve; $\mathrm{PO}$, post-operation

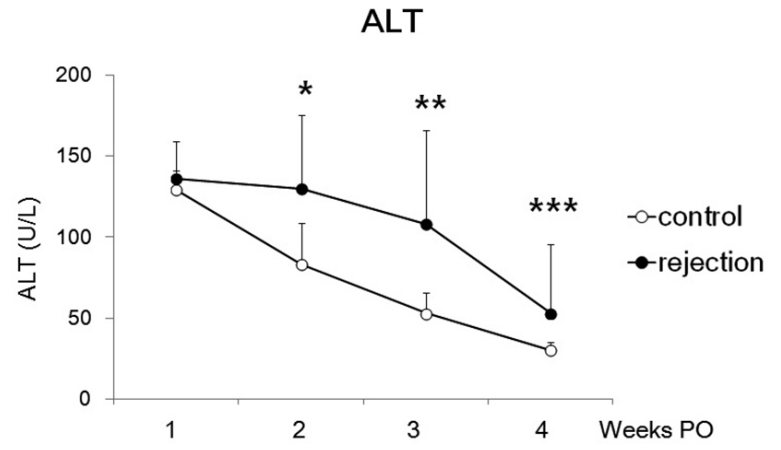

Fig. 1 Serum ALT levels in LT patients with or without acute rejection. Data represent the median + SEM. ${ }^{*} p<0.05 ;{ }^{* \star} p<0.01 ;{ }^{* \star *} p$ $<0.001$.

was considered statistically significant.

\section{RESULTS}

\section{Patients}

Patients with rejection and those without rejection (control group) were similar in age, gender, and types of operation (Table 1). As common in Korea, the majority of transplants were from living donors (over $85 \%$ in both groups). All the donors and recipients were of Korean ethnicity. ABO-incompatible donors accounted for approximately $25 \%$ and $20 \%$ of all living donors in the control group and rejection group, respectively. All patients were given calcineurin inhibitors (approximately $90 \%$ and $85 \%$ tacrolimus and $10 \%$ and $15 \%$ cyclosporin $A$ in the control and rejection groups, respectively) as a part of standard immunosuppressant regimens. There were no significant differences in cold, warm, and total ischemic times or in model of end-stage liver disease (MELD) scores between the two groups. The MELD score comprises serum creatine and serum bilirubin levels, and the international normalized ratio for prothrombin time measurement. The median days from operation to a diagnosis of rejection, or to the date of starting rejection therapy in the case of clinical rejection, was 18 . Seven of the 17 total rejection patients showed clinical rejection.

Liver function was regularly checked using several tests, including measurement of the serum ALT level, which is an important parameter to diagnose rejection. As shown in Fig. 1, ALT concentrations were significantly higher in the rejection group from the $2^{\text {nd }}$ to $4^{\text {th }}$ week PO compared to those of the control group. As acute rejection was diagnosed and treated at an average of 18 days PO (Table 1), serum ALT levels tended to decrease slightly at 4 weeks PO.
Serum IL-10 and IL-17 levels increased at 1-2 weeks PO Several cytokines were measured in the sera from LT patients. We focused specifically on IL-2, IL-4, IL-6, TNF- $\alpha$, IFN- $\gamma$, IL-10, and IL-17A, as they are known to be involved in inflammation, $T$ cell survival and homeostasis, and in the regulation of various effector functions of T cells such as Th1, Th2, regulatory T cells, and Th17. Importantly, all of these cytokines have been shown to be involved in allogeneic immune responses or suggested as biomarkers for rejection. Notably, serum IL-17 concentrations were significantly higher in the rejection patients at 1 week PO (Fig. 2), but decreased after that point. IL-10 levels were significantly higher in the rejection group at 2-3 weeks PO. IL-2 and IL-4 appeared to be increased in the rejection group, but the individual variation was very large. IL- 6 and TNF- $\alpha$ levels were too low to detect in many samples. IFN- $\gamma$ levels appeared to be dynamic and showed high variation. These results suggested that detection of serum IL-10 and IL-17 at proper time points could help to predict the potential for acute rejection.

Serum CXCL10 and CXCL11 levels increased at 2-3 weeks PO CXCR3 ligands were selected for further evaluation, as they have been suggested as biomarkers for graft rejection or dysfunction by many researchers. As shown in Fig. 3, serum CXCL10 and CXCL11 concentrations were significantly increased in the rejection group, whereas CXCL9 concentrations were not. CXCL10 levels increased consistently in the rejection group, and at the $3^{\text {rd }}$ week, the difference was statistically significant. Interestingly, CXCL11 levels dramatically increased from 1 week PO onward. Therefore, CXCL10 and CXCL11 could be diagnostic biomarkers.

\section{Combined detection of IL-10, IL-17, and CXCL10 has} strong predictive power

To verify the potential for the combined use of IL-10, IL-17, CXCL10, and CXCL11 as diagnostic or predictive biomarkers, multivariate logistic regression analysis was performed (Fig. 4 and Table 2). The strongest predictive power was achieved when IL-17 and CXCL10 were assessed at week 1 PO and when IL-10 was assessed at week 2 . The sensitivities of IL-17, IL-10, and CXCL10 were $75 \%, 81 \%$, and $78 \%$, and the specificities were $70 \%, 75 \%$, and $77 \%$, respectively. Multivariate modeling showed that the sensitivity of all three markers together was $94 \%$ and the specificity was $97 \%$ with an AUC value of 0.949 . Unexpectedly, CXCL10 measured at week 1 PO was a better predictive biomarker than CXCL11 measured at week 2 in combination, although the difference in CXCL10 levels between the control and rejection groups was not statistically significant at week 1 . These results demonstrated that serum levels of IL-10, IL-17, and CXCL10 measured at weeks 1 and 2 in combination can predict acute rejection in adult LT patients earlier than diagnosis with conventional methods. 
IL-2

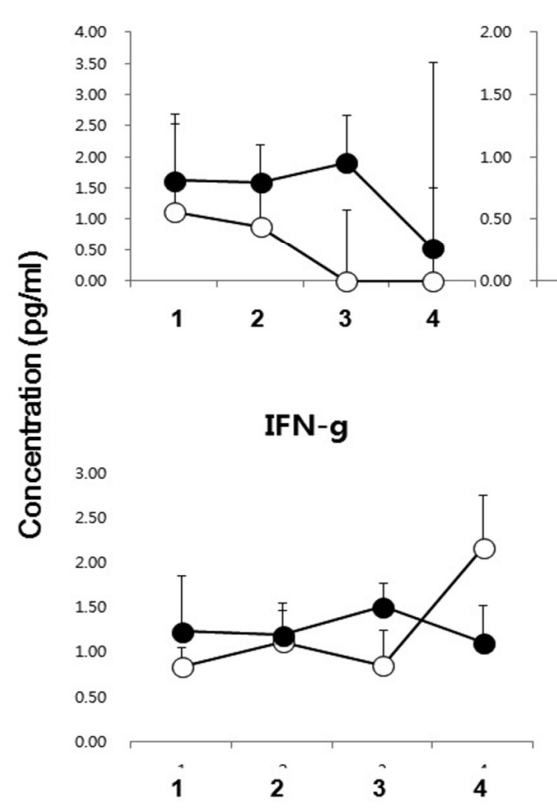

IL-4

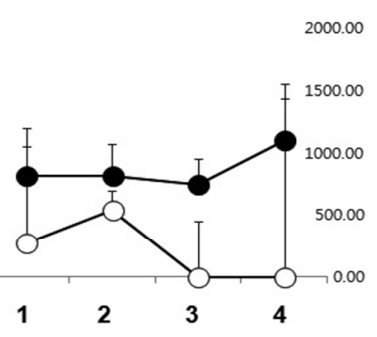

IL-6

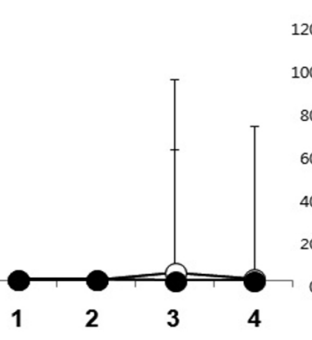

TNF-a

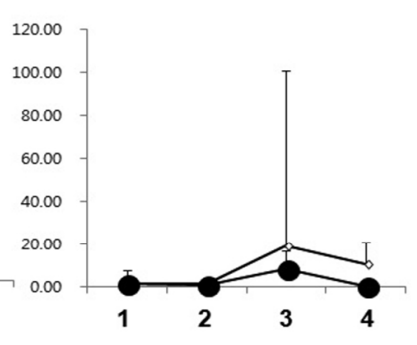

Fig. 2. Serum cytokine levels in LT patients with or without acute rejection. Data represent median $+S E M . N=20,21,16$, and 7 in the control

group; $\mathrm{N}=13,16,15$, and 10 in the rejection group at week $1,2,3$, and 4 , respectively. ${ }^{*} p<0.05$; ${ }^{* *} p<0.01$.
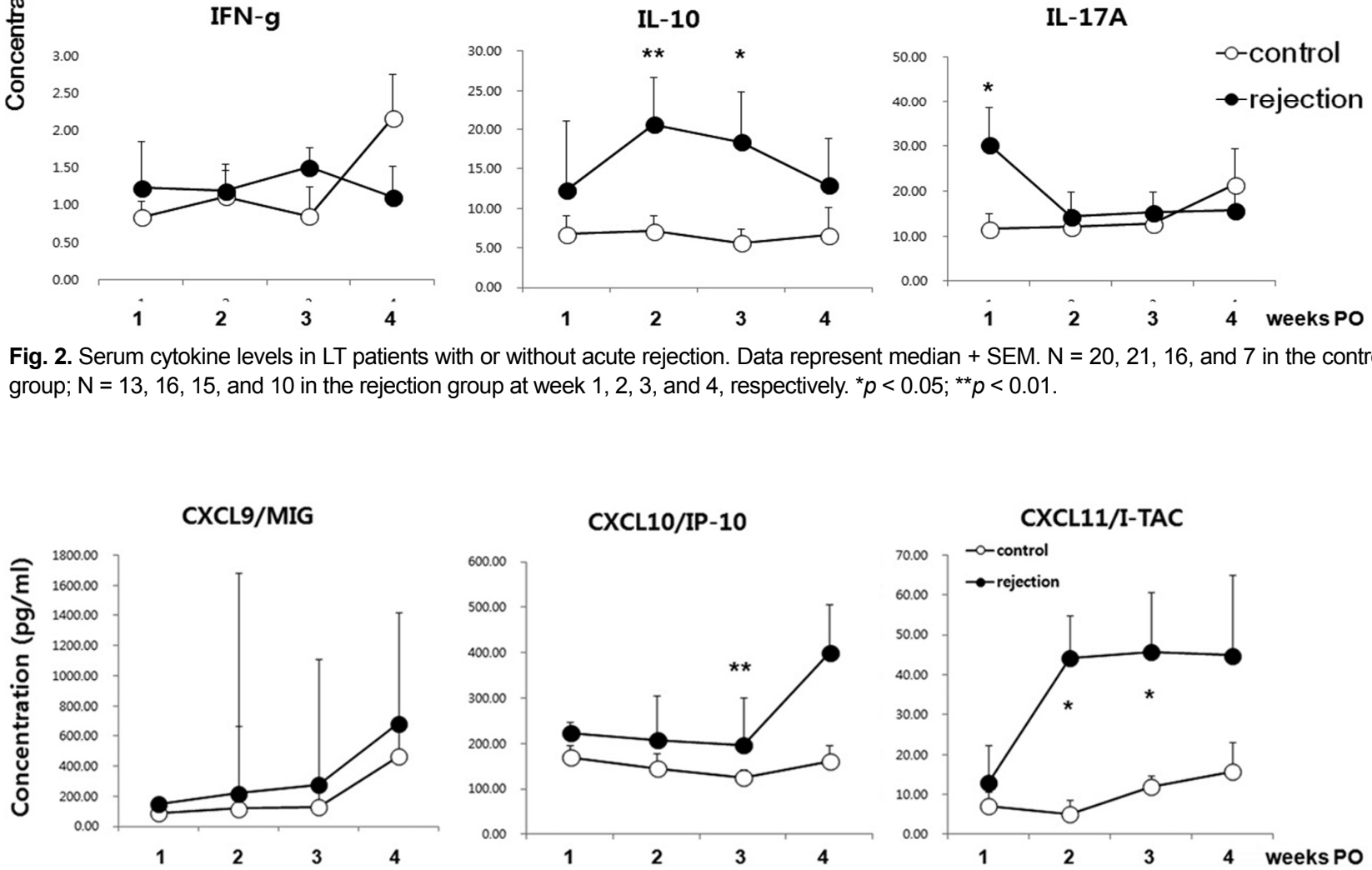

\section{IL-17A}

Fig. 3. Serum CXCR3 ligand levels in LT patients with or without acute rejection. Data represent median + SEM. N = 20, 21, 16, and 7 in the control group; $\mathrm{N}=13,16,15$, and 10 in the rejection group at week $1,2,3$, and 4 , respectively. ${ }^{*} p<0.05 ;{ }^{* *} p<0.01$.

\section{DISCUSSION}

In this study, we suggest the detection of IL-10, IL-17, and CXCL10 in combination as non-invasive predictive biomarkers for acute rejection in LT patients. There was no significant difference in the demographic characteristics of LT patients with and without acute rejection, in any of the parameters compared. This study also supports the results of our previous work showing that $A B O$ incompatibility does not contribute to a higher risk of acute rejection (Song et al., 2014), as the rejection group did not include more patients receiving ABO-incompatible LT.

These results are in line with previous reports demonstrating that IL-17 was increased in the sera from LT patients showing rejection at days 1 and 7 PO (Fabrega et al., 2009). Intracellular IFN- $\gamma$ in activated $\mathrm{T}$ cells was previously shown to increase at
2-4 weeks PO (Millan et al., 2014), whereas IFN- $\gamma$ was not significantly different in the sera of LT patients with and without rejection at same time points in the present study. IL-10 was significantly upregulated in the rejection group in our study. At the time of onset of acute rejection, Th17 cells are significantly higher in the blood of LT patients with acute rejection, while circulating regulatory $T$ cells are decreased (Wang et al., 2014). However, a high frequency of central memory regulatory $T$ cells was detected in LT patients at risk of early acute rejection within the $1^{\text {st }}$ month PO (Boix-Giner et al., 2016). This suggests that the increase in central memory regulatory $T$ cells, rather than naïve regulatory $T$ cells, may have produced the increased IL10 found in this study. In addition, increased Th17 cells and secreted IL-17 may play an important role in acute rejection. Indeed, neutralization of IL-17 with an antibody significantly 
A
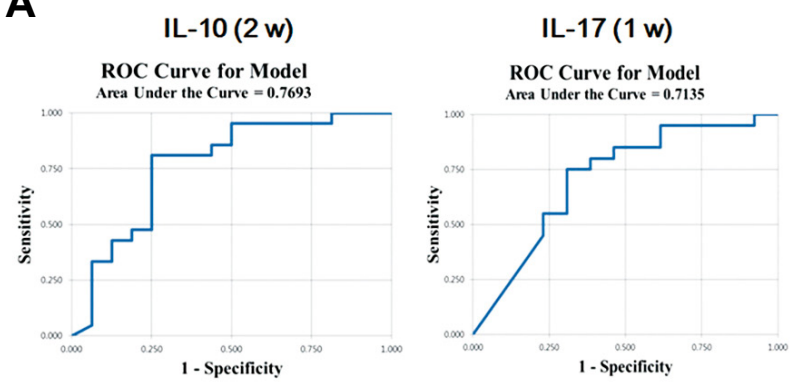

$\operatorname{CXCL10(1~w)~}$
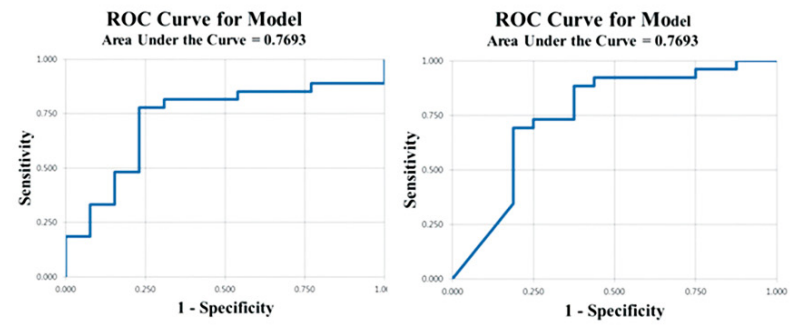

B

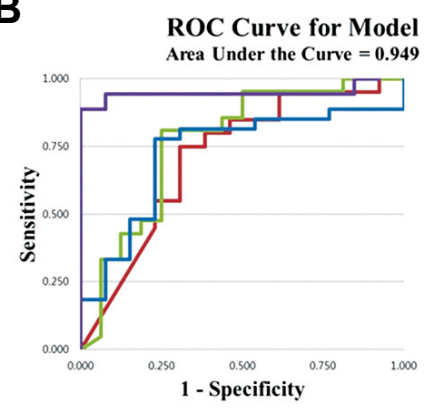

$\operatorname{CXCL11}(2 w)$

1 - Specificity prolonged liver graft survival in rats (Zhou et al., 2015). Notably, increased IL-10 also indicates a high risk for late cytomegalovi rus disease (Krishnan et al., 2010). The present study did not include LT patients with infection; therefore, comparison of serum cytokines and chemokines between LT patients with rejection and with infection may provide more specific differential diagnostic and predictive biomarkers.

IL-2 and IL-4 levels in the sera from the rejection group were slightly higher than those of the control, although these differences were not statistically significant. This weak effect might be related to the relatively small sample size, and therefore a larger cohort study may clarify this issue. Furthermore, IL-6 and TNF- $\alpha$ levels were very low, likely below the detection limits of the assays. One potential reason for the discrepancy in results among studies might be different techniques and affinities of antibodies used to target specific proteins. For example, multiplex cytokine assays are known to be less sensitive than enzyme-linked immunoassays. In addition, the Luminex and Cytometric Bead Array systems have different sensitivities in our experience. Moreover, etiological factors might influence these results. Liver cirrhosis after hepatitis $B$ virus (HBV) infection is prevalent in Korea, which is not the case in Western countries where most previous studies have been conducted. At the time of transplantation, HBV virus titers should be below detectable levels, but the host immune system might be biased in cases of chronic infection. In addition, the majority of LT surgeries in Korea involve living donors, which are usually associated with a lower ischemic time. Thus, ischemia reperfusion injury and consequent inflammation could be milder in Korean LT patients.

Serum CXCL10 and CXCL11 appear to be more potent biomarkers than CXCL9 for acute rejection in LT, according to the results of the present study. However, CXCL11 has been relatively less investigated than CXCL9 and CXCL10 in previous studies. Serum CXCL9 and CXCL10 were found to be significantly higher in LT patients with early allograft dysfunction on day 1 PO, but not subsequently (Friedman et al., 2012). The
Fig. 4. Predicting the capacity for acute rejection. Receiver operating characteristic (ROC) curve analysis was performed to define the area under the curve (AUC), sensitivity, and specificity for IL-10 at week 2, IL-17 at week 1 , and CXCL10 at week 1 PO. (A) Predictive capacity of each parameter. (B) Multivariate analysis of IL10, IL-17, and CXCL10. main cause of early allograft dysfunction is thought to be ischemia reperfusion injury, whereas the current study focused on acute rejection, which occurs later than ischemia reperfusion injury. CXCR3 is expressed in activated T cells, which plays a main role in acute cellular rejection. Therefore, increased CXCR3 ligands could promote activated T cell migration and rejection. CXCR3 and IL-10 were found to be down-regulated in the proliferative phase during liver regeneration in rats $(X u$ et al., 2011), suggesting that up-regulated CXCR ligands and IL10 might have regulatory roles in liver regeneration, thereby delaying graft accommodation.

As IL-17 was found to be up-regulated at 1 week $P O$, it might be interesting to assess CCL20, a CCR6 ligand, which plays a key role in the migration of Th17 cells (Kim, 2009). A previous report showed that liver graft strongly upregulated CCL20 within $1 \mathrm{~h}$ after ischemia reperfusion injury (Ingelsten et al., 2011). In addition, loss of HERC5 expression in patients with recurrent hepatocellular carcinoma (HCC) post-LT has been suggested to drive the up-regulation of CCL20 in HCC cells (Xue et al., 2015). It should be noted that ischemic times were not different between the rejection and control groups in our study, and transplant patients with $\mathrm{HCC}$ are relatively rare in Korea. To our knowledge, CCL20 has not been suggested as a biomarker for graft rejection, but it would be interesting to evaluate its potential value.

As mentioned above, some cytokines and chemokines are involved in not only the rejection process but also in other inflammatory and immunological events; hence, it would be useful to combine multiple biomarkers to achieve more accurate diagnosis and prediction. Therefore, in this study, multivariate modeling was performed with IL-10, IL-17, CXCL10, and CXCL11 at 1-2 weeks PO. To our surprise, CXCL10 at week 1 $\mathrm{PO}$ emerged as a better predictive biomarker than CXCL11 at week 2, implying that the difference in serum CXCL10 levels might actually be statistically significant in a larger cohort. The strongest prediction rate was achieved when IL-10 at 2 weeks PO, and IL-17 and CXCL10 at 1 week PO were combined in 
the analysis. With these three factors, acute rejection could be predicted in 2 weeks, which is days earlier than the average diagnosis time obtained with conventional methods. Earlier diagnosis could provide a better opportunity to treat rejection and consequently preserve graft function. These candidate biomarkers should also be evaluated at earlier time points such as at 1-7 days $\mathrm{PO}$ or even peri-operationally.

In conclusion, the combined detection of serum IL-10, IL-17, and CXCL10 can predict acute rejection following adult liver transplantation. However, the results must be confirmed in larger cohort studies.

\section{ACKNOWLEDGMENTS}

We are grateful to all of the liver transplant staff at AMC, the NK Lab members, and the flow cytometry core facility at Asan Institute for Life Sciences. We thank Prof. J. B. Lee for statistical analysis. This research was supported by the Basic Science Research Program through the National Research Foundation of Korea (NRF) funded by the Ministry of Science, ICT and Future Planning (Grant No. 2015R1A2A2A04007141) and by grants from the Asan Institute for Life Sciences, Asan Medical Center (Grant No 12/13-542).

\section{REFERENCES}

Berres, M.L., Trautwein, C., Schmeding, M., Eurich, D., Tacke, F., Bahra, M., Neuhaus, P., Neumann, U.P., and Wasmuth, H.E. (2011). Serum chemokine CXC ligand 10 (CXCL10) predicts fibrosis progression after liver transplantation for hepatitis C infection. Hepatology 53, 596-603.

Boix-Giner, F., Millan, O., San Segundo, D., Munoz-Cacho, P., Mancebo, E., Llorente, S., Rafael-Valdivia, L., Rimola, A., Fabrega, E., Mrowiec, A., et al. (2016). High frequency of central memory regulatory $T$ cells allows detection of liver recipients at risk of early acute rejection within the first month after transplantation. Int. Immunol. 28, 55-64.

Capece, T., and Kim, M. (2016). The role of lymphatic Niches in T cell differentiation. Mol. Cells 39, 515-523

Fabrega, E., Lopez-Hoyos, M., San Segundo, D., Casafont, F., and Pons-Romero, F. (2009). Changes in the serum levels of interleukin-17/interleukin-23 during acute rejection in liver transplantation. Liver Transpl. 15, 629-633.

Friedman, B.H., Wolf, J.H., Wang, L., Putt, M.E., Shaked, A., Christie, J.D., Hancock, W.W., and Olthoff, K.M. (2012). Serum cytokine profiles associated with early allograft dysfunction in patients undergoing liver transplantation. Liver Transpl. 18, 166-176.

Huang, W.H., Yan, Y., Li, J., De Boer, B., House, A.K., and Bishop, G.A. (2003). A short course of mycophenolate immunosuppression inhibits rejection, but not tolerance, of rat liver allografts in association with inhibition of interleukin-4 and alloantibody responses. Transplantation 76, 1159-1165.

Ingelsten, M., Karlsson-Parra, A., Granqvist, A.B., Molne, J., Olausson, M., Haraldsson, B., and Nystrom, J. (2011). Postischemic inflammatory response in an auxiliary liver graft predicts renal graft outcome in sensitized patients. Transplantation 91, 888-894.

Karakhanova, S., Oweira, H., Steinmeyer, B., Sachsenmaier, M., Jung, G., Elhadedy, H., Schmidt, J., Hartwig, W., Bazhin A. V., and Werner, J. (2016). Interferon-gamma, interleukin-10 and interferon- inducible protein 10 (CXCL10) as serum biomarkers for the early allograft dysfunction after liver transplantation. Transpl. Immunol. 34, 14-24.

Kim, C.H. (2009). Migration and function of Th17 cells. Inflamm Allergy Drug Targets 8, 221-228.

Krishnan, A., Zhou, W., Lacey, S.F., Limaye, A.P., Diamond, D.J. and La Rosa, C. (2010). Programmed death-1 receptor and interleukin-10 in liver transplant recipients at high risk for late cytomegalovirus disease. Transpl. Infect Dis. 12, 363-370.

Lee, B., Oh, C.K., Kim, M.S., Kim, J.H., Kim, S.J., Kim, H.S., and Shin, G.T. (2012). Cytokine gene expression in peripheral blood mononuclear cells during acute renal allograft rejection. Transplant Proc. 44, 236-240.

Millan, O., Rafael-Valdivia, L., Torrademe, E., Lopez, A., Fortuna, V., Sanchez-Cabus, S., Lopez-Pua, Y., Rimola, A., and Brunet, M. (2013). Intracellular IFN-gamma and IL-2 expression monitoring as surrogate markers of the risk of acute rejection and personal drug response in de novo liver transplant recipients. Cytokine 61 , 556-564.

Millan, O., Rafael-Valdivia, L., San Segundo, D., Boix, F., CastroPanete, M.J., Lopez-Hoyos, M., Muro, M., Valero-Hervas, D., Rimola, A., Navasa, M., et al. (2014). Should IFN-gamma, IL-17 and IL-2 be considered predictive biomarkers of acute rejection in liver and kidney transplant? Results of a multicentric study. Clin. Immunol. 154, 141-154.

Nakagiri, T., Inoue, M., Minami, M., Shintani, Y., and Okumura, M. (2012). Immunology mini-review: the basics of $\mathrm{T}(\mathrm{H}) 17$ and interleukin-6 in transplantation. Transplant Proc. 44, 1035-1040.

Raschzok, N., Reutzel-Selke, A., Schmuck, R.B., Morgul, M.H., Gauger, U., Prabowo, K.A., Tannus, L.M., Leder, A., Struecker, B., Boas-Knoop, S., et al. (2015). CD44 and CXCL9 serum protein levels predict the risk of clinically significant allograft rejection after liver transplantation. Liver Transpl. 21, 1195-1207.

Song, G.W., Lee, S.G., Hwang, S., Kim, K.H., Ahn, C.S., Moon, D.B. Ha, T.Y., Jung, D.H., Park, G.C., Kang, S.H., et al. (2014). Biliary stricture is the only concern in ABO-incompatible adult living donor liver transplantation in the rituximab era. J. Hepatol. 61, 575-582.

Sreekumar, R., Rasmussen, D.L., Wiesner, R.H., and Charlton, M.R. (2002). Differential allograft gene expression in acute cellular rejection and recurrence of hepatitis $C$ after liver transplantation. Liver Transpl. 8, 814-821.

Tannapfel, A., Geissler, F., Witzigmann, H., Hauss, J., and Wittekind C. (2001). Analysis of liver allograft rejection related genes using cDNA-microarrays in liver allograft specimen. Transplant Proc. 33 3283-3284.

Wang, Y., Zhang, M., Liu, Z.W., Ren, W.G., Shi, Y.C., Sun, Y.L., Wang, H.B., Jin, L., Wang, F.S., and Shi, M. (2014). The ratio of circulating regulatory $T$ cells (Tregs)/Th17 cells is associated with acute allograft rejection in liver transplantation. PLoS One 9 e112135.

Xu, C., Chen, X., Chang, C., Wang, G., Wang, W., Zhang, L., Zhu, Q., Wang, L., and Zhang, F. (2011). Analysis of gene expression profiles of liver stellate cells during liver regeneration in rats. Mol. Cells 31, 17-23.

Xue, F., Higgs, B.W., Huang, J., Morehouse, C., Zhu, W., Yao, X. Brohawn, P., Xiao, Z., Sebastian, Y., Liu, Z., et al. (2015). HERC5 is a prognostic biomarker for post-liver transplant recurrent human hepatocellular carcinoma. J. Transl. Med. 13, 379.

Zhou, Y., Yang, X., Zhang, H., and Jiang, J. (2015). The roles of T helper type 17/regulatory $T$ cells in acute rejection after liver transplantation in rats. Transplantation 99, 1126-1131. 